\title{
Influence of the shaping effect on hardness homogeneity by Vickers indentation analysis
}

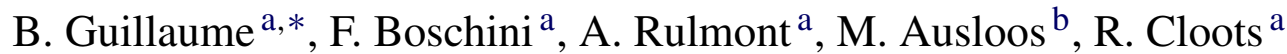 \\ a Laboratoire de Chimie Inorganique Structurale, SUPRATECS, Department of Chemistry, Chemistry Inst. B6, \\ University of Liège, Sart-Tilman, B-4000 Liège, Belgium \\ b Physique Statistique et des Matériaux, SUPRATECS, Department of Physics, Physics Inst. B5, \\ University of Liège, Sart-Tilman, B-4000 Liège, Belgium
}

Received 29 May 2005; received in revised form 16 August 2005; accepted 19 August 2005

Available online 21 October 2005

\begin{abstract}
In this study, indentation technique (Vickers indentation) has been unconventionally used to evaluate the homogeneity of barium zirconate ceramic samples which have been shaped through different routes. Statistical tools have been used to estimate the correlation which can be established between heterogeneities within the samples and their shaping ways.
\end{abstract}

(c) 2005 Elsevier Ltd. All rights reserved.

Keywords: Hardness; Defects; Perovskites; Statistical analysis

\section{Introduction}

Mechanical properties of materials are usually determined with the help of classical bending or tensile tests; most of the time samples with specific forms are required. It is not sometimes possible to produce such a specific sample in order to evaluate its mechanical properties. Indeed, some materials are brittle, rare or expensive such that the machining of samples is often delicate or inconceivable. Indentation tests are then considered to be suitable tools to evaluate mechanical properties of materials difficult to shape. Depending on the indenter geometry and the load used, indentations are about a few nanometres in depth and a few micrometers wide. Indentation tests are thus a local probe but they can be used for small size samples.

As a result, several measurements and a statistical treatment are needed in order to characterize with precision the investigated material.

Based on the same approach, this technique should be interesting for examining possible heterogeneity within a given material.

\footnotetext{
* Corresponding author. Tel.: +32 436635 32; fax: +32 43663413 .

E-mail address: b.guillaume@ulg.ac.be (B. Guillaume).
}

The indentation techniques give only information about the mechanical properties of the sample surface which must be previously well polished.

Mechanical properties for coating layers ${ }^{1}$ or laminated composites $^{2}$ are determined by indentation tests. Among mechanical properties which can be deduced from indentation tests, the hardness $(H)$ and the fracture toughness $\left(K_{\mathrm{c}}\right)$ are the most popular ones. ${ }^{3,4}$

It is also possible to measure the thermal shock behaviour of brittle refractory materials using an indentation-quench method. ${ }^{5-8}$

To evaluate the thermal shock behaviour, samples containing Vickers indentations are submitted to quenching and the growth of the radial cracks is analysed. It is also possible, under certain conditions, to determine the Young's modulus by Knoop indentation, ${ }^{4}$ as well as a relationship between hardness and intrinsic mechanical properties. ${ }^{9}$

In summary, indentation techniques are suitable to determine surface mechanical properties of samples which cannot be manufactured in specific size and shape required when classical tests are used (bending or tensile tests).

In this report, Vickers indentations (and the associated hardness values) have been unconventionally used to evaluate (by mapping) the homogeneity of a given sample which has been shaped by different methods. 
Table 1

Relative densities of green and sintered bodies

\begin{tabular}{|c|c|c|c|c|}
\hline & Green density (\% TD) & Sintered density (\% TD) & Sintered open porosity $(\%)$ & Sintered closed porosity (\%) \\
\hline SLIP & 50 & 60 & 39 & 0 \\
\hline UNIP & 56 & 69 & 30 & 1 \\
\hline ISOP & 55 & 69 & 30 & 1 \\
\hline
\end{tabular}

Data recorded from Vickers indentations made on samples shaped by different methods have been compared by using successively elementary statistical analysis, mappings of hardness values for each samples and Weibull distributions. Moreover, non-parametric Kruskal-Wallis test has been conducted to check if the shaping process influences significantly the average hardness value.

\section{Experimental}

A commercial barium zirconate powder (Alfa-Aesar, 99\%), which has a median particle size around $1.6 \mu \mathrm{m}(d=0.5)$, was milled with a planetary grinder (Retsch PM 400\2) using tungsten carbide jar and $3 \mathrm{~mm} \mathrm{MgO}$-stabilized zirconia's balls (Tosoh). After mechanical grinding, a median particle size of $0.6 \mu \mathrm{m}(d=0.5)$ was obtained. ${ }^{10}$

Three different techniques were used to shape the samples

(i) the slip casting-the so-called SLIP sample;A powder/water suspension made of (i) solid contents (30 vol.\%; $72 \mathrm{wt} . \%)$ and (ii) dispersing agent (1 wt.\% (PMAADolapix CE64, Zschimmer \& Schwarz)) was slip casted in plaster of Paris cylindrical moulds. ${ }^{11}$

(ii) the uniaxial pressing-the so-called UNIP sample;This second sample was uniaxially pressed. Powder was slipped into a cylindrical steel mould and a pressure of $200 \mathrm{bar}$ was applied during $2 \mathrm{~min}$. The mould inner surface was lubricated with the help of a magnesium stearate/acetone solution.

(iii) the isostatic pressing-the so-called ISOP sample;The powder was poured into a cylindrical PVC mould and pressed at 2000 bar during $2 \mathrm{~min}$.

According to previous study of barium zirconate sintering processing, ${ }^{10}$ it has been possible to select a specific heating program to obtain a relatively porous body.

The samples have been then sintered in air by using the following heating program: isotherm temperature, $1350^{\circ} \mathrm{C}$; heating rate, $1^{\circ} \mathrm{C} / \mathrm{min}$; soaking time, $2 \mathrm{~h}$; cooling rate, $10^{\circ} \mathrm{C} / \mathrm{min}$.
Notice that it is preferable to perform indentation on a porous ceramic in order to not observe 'piling up' or 'sinking-in' of the material around the Vickers indentation. Indeed, during indentation experiments, the material around the contact area tends to deform upwards or downwards where the load is applied. Such surface deformation modes influence the hardness measurements since the true contact area between the indenter and the specimen increases in the case where pilling-up predominates, while it decreases in the event that sinking-in occurs. ${ }^{12,13}$ Piling up can be attributed to the residual stresses produced during impression. Pressure produced during indentation is transferred to neighbour cells, but more intensely to those immediately below the indenter. ${ }^{12}$ As a result, the indentation picture focus is not easily made.

The densities of the green pellets were calculated from the sample mass and dimension.

The green body density values are summarised in Table 1 as well as the relative densities, open porosity and closed porosity of sintered samples measured using the Archimede method with $n$-butanol as solvent.

Relative densities were based on a $6.242 \mathrm{~g} / \mathrm{cm}^{3}$ theoretical density which has been calculated from $\mathrm{BaZrO}_{3}$ crystallographic data.

Sintered bodies have been fixed in epoxy resin and then polished with silicon carbide paper and diamond paste until a perfect polished surface is obtained. To improve the contrast between the indentation and the surface and to facilitate the picture focus, a thin layer of silver $(10 \mathrm{~nm})$ was coated on the polished surface sample with the help of a sputter-coating machine. Preliminary study revealed that a thin silver layer coating does not influence significantly the hardness of the sample.

The dimensions of green, sintered and polishing samples are summarised in Table 2.

Vickers indentations are realized with an Instron WilsonWolpert Tukon 2100B Hardness Tester. This tester uses state of the art closed loop load cell technology to apply the test forces. This ensures that the loading force is constant during the test dwell time.

The Vickers indenter is a diamond square-based pyramid with an angle of $136^{\circ}$ between faces. The depth of the indentation is

Table 2

Dimension of green, sintered and polishing bodies

\begin{tabular}{lllllll}
\hline & $\begin{array}{l}\text { Green diameter } \\
(\mathrm{mm})\end{array}$ & $\begin{array}{l}\text { Green thickness } \\
(\mathrm{mm})\end{array}$ & $\begin{array}{l}\text { Sintered diameter } \\
(\mathrm{mm})\end{array}$ & Sintered thickness $(\mathrm{mm})$ & $\begin{array}{l}\text { Polished diameter } \\
(\mathrm{mm})\end{array}$ & $\begin{array}{l}\text { Polished thickness } \\
(\mathrm{mm})\end{array}$ \\
\hline SLIP & 20 & 10 & 16 & 8 & 16 & 4 \\
UNIP & 33 & 12 & 22 & 8 & 22 & 4 \\
ISOP & 32 & 14 & 23 & 10 & 23 & 5 \\
\hline
\end{tabular}


Table 3

Descriptive basic statistics for the investigated bodies

\begin{tabular}{lccr}
\hline & SLIP & \multicolumn{1}{c}{ ISOP } & \multicolumn{1}{c}{ UNIP } \\
\hline Indentations number & 740 & 1390 & 1240 \\
Minimum (GPa) & 2.363 & 2.893 & 0.469 \\
Maximum (GPa) & 5.423 & 6.031 & 7.512 \\
Range (GPa) & 3.060 & 3.138 & 7.043 \\
Mean (GPa) & 4.548 & 5.211 & 5.120 \\
Standard deviation $(\mathrm{GPa})$ & 0.406 & 0.284 & 0.688 \\
R.S.D. $(\%)$ & 8.924 & 5.441 & 13.443 \\
\hline
\end{tabular}

about one-seventh of its diagonal length. The Vickers hardness number $\mathrm{Hv}$, in $\mathrm{kg} \mathrm{F} / \mathrm{mm}^{2}$, is computed from the Eq. (1) where $P$, $A_{\mathrm{S}}, d, \alpha$ are, respectively, the test force (in $\mathrm{kg} \mathrm{F}$ ), the surface area of indentation (in $\mathrm{mm}^{2}$ ), the mean diagonal of indentation (in $\mathrm{mm}$ ) and the face angle of indenter $\left(136^{\circ}\right)$. Usually, Vickers hardness values are expressed in Vickers units $(\mathrm{Hv})$ or in Pascal $(\mathrm{Pa})$ according to the international system units $(1 \mathrm{Hv}=9.807 \mathrm{MPa})$.

$\mathrm{Hv}=\frac{1000 P}{A_{\mathrm{s}}}=2000 \frac{P \sin \alpha / 2}{d^{2}}=1.854 \frac{P}{d^{2}}$

In this study, the loading force was $0.5 \mathrm{~kg} \mathrm{~F}(4.905 \mathrm{~N})$ and the dwell time $10 \mathrm{~s}$. The average of indentation depth is $5 \mu \mathrm{m} .{ }^{14}$ For the three different samples, the polished surface has been indented with a $500 \mu \mathrm{m}$ step.

\section{Results and discussion}

\subsection{Data analyses}

Table 3 summarises descriptive statistics for the three investigated samples.

Plots of the three hardness value distributions are presented in Fig. 1.

Hardness values associated to ISOP and UNIP samples are larger than the one associated to the SLIP sample. The mean hardness value is relatively identical for the pressed samples $(\sim 5.15 \mathrm{GPa})$ and, of course, a lower average hardness value is

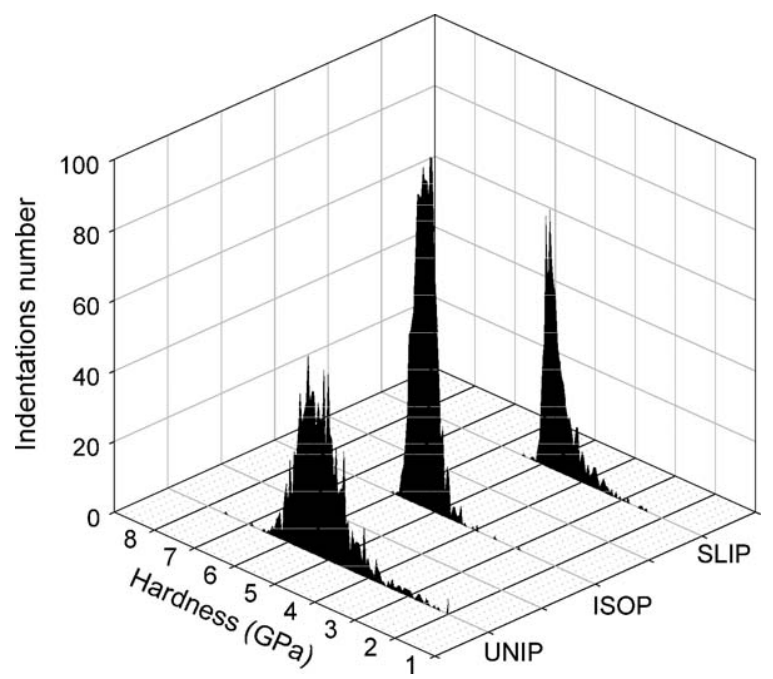

Fig. 1. Distribution of recorded hardness values for each samples.

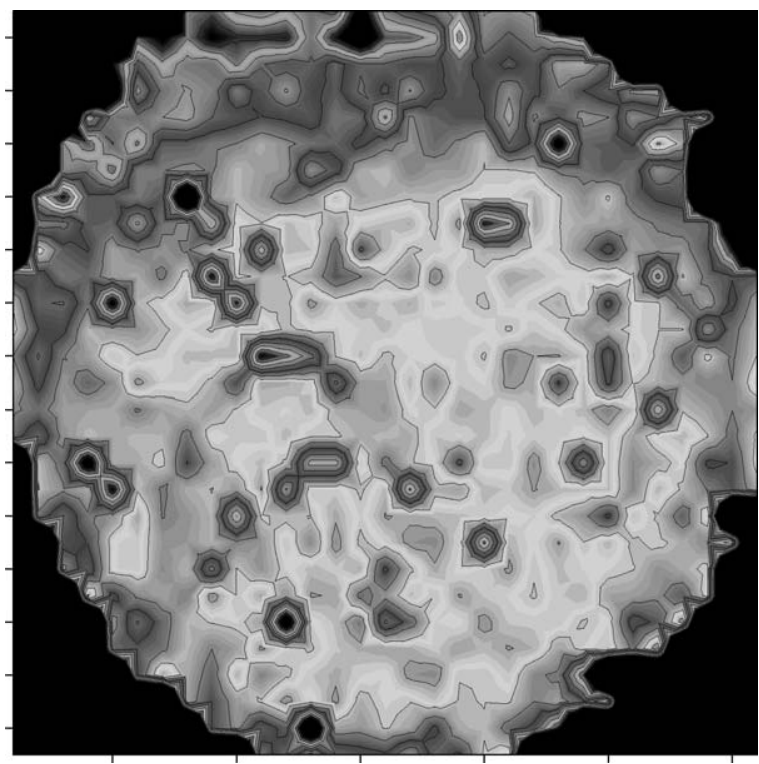

Fig. 2. Mapping of $k$ values for SLIP sample.

recorded for the SLIP sample. This is due to the fact that the SLIP sample is less dense than ISOP and UNIP ones (Table 1).

Extreme values (respectively, 0.469 and $7.512 \mathrm{GPa}$ as minimum and maximum hardness values) and the widest distribution are found for the UNIP sample.

It is usual to use relative values as objective criteria to compare distributions. The relative standard deviation (R.S.D.) is defined as the ratio between the standard deviation and the mean value. A small/high R.S.D. value means that the value distribution is narrow/large.

From Table 3, R.S.D. value comparisons indicate that the hardness value distribution is the lowest/largest for ISOP/UNIP sample.

\subsection{Analysis of hardness value mapping}

Considering that the density, and so hardness values, for the SLIP sample are lower than for ISOP and UNIP samples, to compare objectively the three mappings, it is necessary to create new normalized data.

Within a distribution $i$, a given $j$ hardness value $\left(\mathrm{Hv}_{i, j}\right)$ can be expressed with the help of the mean value $\left(\overline{\mathrm{Hv}_{i}}\right)$ and the standard deviation $\left(\sigma_{i}\right)$ of its $i$ distribution through (Eq. (2)):

$\mathrm{Hv}_{i, j}=\overline{\mathrm{Hv}_{i}}+k_{j} . \sigma_{i}$

It is then possible to create a dimensionless normal variable $\left(k_{j}\right)$ devoid of unities, which has zero mean and unitary standard deviation (Eq. (3)).

$k_{j}=\frac{\mathrm{Hv}_{i, j}-\overline{\mathrm{Hv}_{i}}}{\sigma_{i}}$

In this way, it is possible to meaningfully compare the (hardness) homogeneity within different samples which have not exactly the same hardness mean values. 


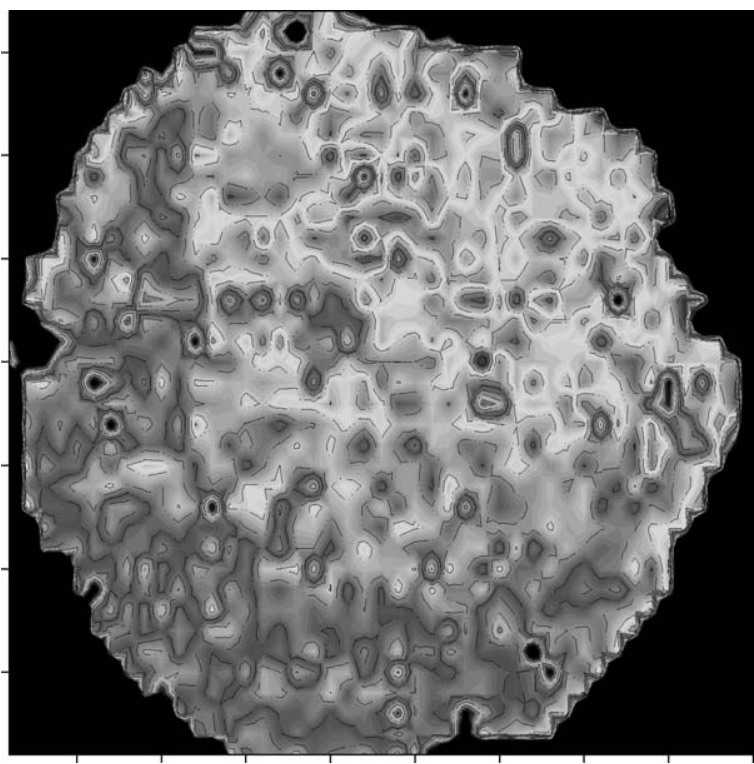

Fig. 3. Mapping of $k$ values for UNIP sample.

Figs. 2-4 represent mapping set of these $k$ values for SLIP, UNIP, ISOP samples, respectively. In these figures, small/high absolute values of $k$ are, respectively, represented by a light/dark grey.

From the analysis of the three samples mapping, it appears that some area are harder than others.

ISOP and UNIP samples present gradient of hardness values across investigated samples, while the SLIP sample seems to be more homogenous.

Curiously this gradient is particularly marked in the ISOP sample while it presents the lowest R.S.D. value.

The pressing step thus induces a density gradient inside the green body. Of course, this gradient is also present in the sintered

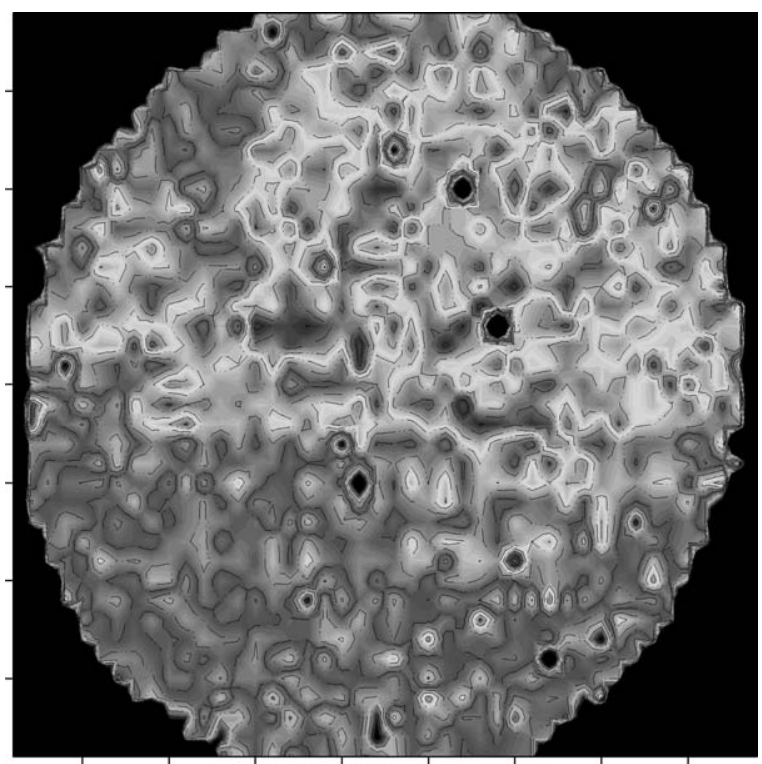

Fig. 4. Mapping of $k$ values for ISOP sample. body and permits to understand why some areas are harder than others inside a pressing sample.

Several experimental results have been reported on the study of granule properties and how they interact during the pressing step. ${ }^{15,16}$

Onoda ${ }^{17}$ has studied the influence of granule properties by comparing the compaction of 'hard', 'medium' and 'soft' granules. It appears that soft granules produce high-density compacts with large-density gradients. In fact, soft granules deform readily under the pressure but, if too soft, will not rearrange sufficiently at low pressure to eliminate large packing defects. In the former case, large residual voids remain after compaction.

\subsection{Detection of outliers}

Before to lead Weibull analysis, it is suitable to detect if outlier are present in the data or not.

Considering that data do not follow normal distribution, the presence of potential outliers has been checked by a nonparametric statistical rank test.

A value, which is not included in the range defined by the following equation (Eq. (4)), has been considered as outlier one. In this one, $Q_{i}$ represents the quartiles of investigated distribution. A quartile is any of the three values, which divide the investigated data into four equal parts, so that each part represents one-fourth of the population.

$X_{\text {OUT }} \notin\left[Q_{1}-1.5\left(Q_{3}-Q_{1}\right), Q_{3}+1.5\left(Q_{3}-Q_{1}\right)\right]$

It is suitable to detect outliers because the presence of them could be embarrassing during the fitting steps. Detected outliers have been removed from initial data distributions to conduct Weibull analysis and Kruskall-Wallis test.

\subsection{Weibull distribution}

One can also assume that the statistical variability of the indentation hardness within one sample may be described by a Weibull distribution function (Eq. (5))

$F\left(\sigma_{\mathrm{c}}\right)=1-\exp \left\{-\left(\frac{\sigma_{\mathrm{c}}}{\sigma_{0}}\right)^{m}\right\}$

Indeed, to evaluate the homogeneity of the samples, it is suitable to use the Weibull statistics. ${ }^{18}$ The Weibull distribution function $F\left(\sigma_{\mathrm{c}}\right)$ is commonly used to assess the reliability of the materials against mechanical disturbance, i.e. indentation. $\sigma_{\mathrm{c}}$ represents a critical value of a characteristic stress; $\sigma_{0}$ and $m$ are a scale parameter and the Weibull modulus, respectively. ${ }^{19,20}$

The Weibull modulus $(m)$ can be considered as a variability indicator. A sample exhibiting large/low $m$ value has high/low reliability against mechanical disturbance. Typical values of $m$ are 5 for blackboard chalk, 10 for engineering ceramics and 100 for steels. ${ }^{21}$

In our study, the mechanical disturbance is a Vickers indentation and the corresponding hardness values $\left(\mathrm{Hv}_{i, j}\right)$ is assimilated to the variable $\sigma_{\mathrm{c}}$. A change of variables and an adequate logarithmic transformation permits to rewrite the general Weibull 


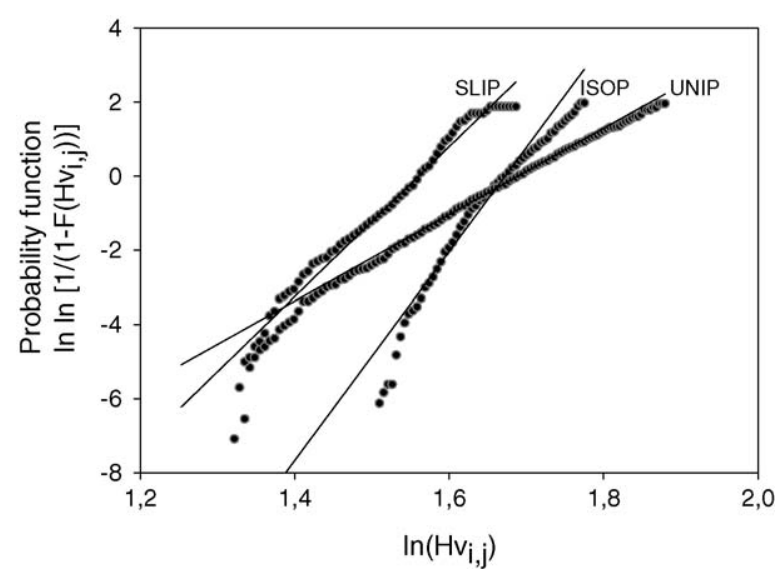

Fig. 5. Weibull plot.

distribution function as follows (Eq. (6)):

$\ln \ln \left(\frac{1}{1-F\left(\operatorname{Hv}_{i, j}\right)}\right)=m \ln \left(\operatorname{Hv}_{i, j}\right)-m \ln \left(\overline{\operatorname{Hv}_{i}}\right)$

It is now possible to deduce the mechanical parameters with the help of a least square linear regression model (Fig. 5).

The Fig. 5 shows the Weibull plot for the measured hardness data.

Weibull modulus $(m)$, parameter scale $\left(\sigma_{0}=\overline{\mathrm{Hv}}{ }_{i}\right)$ for each samples and linear regression coefficient $\left(R^{2}\right)$ are given in Table 4.

It appears that the $R^{2}$ coefficient relating to ISOP data's regression is rather small. This might imply that the statistical variability of measured indentation hardness for each sample may not be described by a Weibull distribution. Whence the null hypothesis $\left(\mathrm{H}_{0}\right.$ - variability is NOT described by a Weibull distribution function) has been tested for the three distributions.

Pearson $\chi^{2}$ tests have thus been conducted, using the estimated $m$ and $\overline{\mathrm{Hv}_{i}}$, with the measured data set according to standard statistical theory. $\chi^{2}$ tests reveal that a Weibull distribution can be accepted at a very high confidence level for each distribution $(p$-values $<0.001)$.

Weibull parameters $(m)$ reveal that $I S O P$ sample assesses a more important reliability against the indentation disturbance than the two other samples (SLIP and UNIP). This confirms the pre-analysis based on the R.S.D. value study (Table 3).

\subsection{Statistical analysis}

As revealed in the previous sections, it seems that shaping way have an influence on the distribution broad of studied samples. At present, it is suitable to statistically verify if those

Table 4

Linear regression coefficient (Weibull parameters) of the investigated bodies

\begin{tabular}{lllc}
\hline & SLIP & ISOP & UNIP \\
\hline$m$ & 20.2 & 28.1 & 11.6 \\
$\overline{\mathrm{Hv}}(\mathrm{GPa})$ & 4.62 & 5.22 & 5.2 \\
$R^{2}$ & 0.97 & 0.87 & 0.99 \\
\hline
\end{tabular}

distributions broads could have an influence on the investigated samples global hardness values. To do that, Kruskall-Wallis tests have been done on the hardness recorded values for each studied sample.

From Kruskall-Wallis test, which is presented in Annexe A, it appears that the median hardness value is not influenced by the shaping way. In others words, even if only few indentations are realized on each sample (instead of mapping), the global hardness value does not be influenced by the shaping way.

\section{Conclusions}

Vickers indentations have been realized on three samples which have been shaped by different way.

Indentation technique is a possible tool to probe locally a sample and thus to determine its compositional heterogeneity.

Data and Weibull analyses reveal that shaping way influences the hardness homogeneity inside samples.

However, Kruskall-Wallis test suggests to not considering as influent the shaping way on the hardness median values.

\section{Acknowledgements}

This work has been financially supported by the Belgium French Community (Communauté Française de Belgique) within the framework of a Concerted Research Action on Granular Materials no 02/07-293.

\section{Annexe A. Analysis of variances by ranks; Kruskall-Wallis test}

Usually, to compare different distributions between them, it is a usual practice to realize analysis of variance (ANOVA) study. Several concepts concerning ANOVA can be found in reference. ${ }^{22}$ In few statistical words, ANOVA tests if the global variability between distributions is greater than global variability within these distributions. It is then possible to check if means across the distributions are different in magnitude, and then if those distributions are independent from the others.

Among the assumptions required to conduct ANOVA, data normality is an important one.

Unfortunately, as mentioned above, data recorded for each sample do not follow normal distribution. It is then necessary to use a non-parametric test to compare different distributions between them.

The Kruskal-Wallis test offers a non-parametric alternative to the ANOVA. ${ }^{23}$

Exhaustive information about Kruskall-Wallis test can be found in reference. ${ }^{24}$

In a few words, this test compares different distributions $(k)$ and tests the (null) hypothesis $\mathrm{H}_{0}$ that the investigated distributions (do not) come from the same distribution or from distribution with the same median value.

Eq. (7) represents the hypothesis $\mathrm{H}$; in this one, $n_{j}$ is the number of observations in group $j, N$ is the total sample size, 
$\overline{R_{j}}$ is the average of the ranks for a done group $j$ and $\bar{R}$ is the average of all the ranks.

$\mathrm{H}=\frac{12 \sum_{k=1}\left[\overline{R_{j}}-\bar{R}\right]^{2}}{N(N+1)}$

If null hypothesis $\left(\mathrm{H}_{0}\right)$ is verified, the distribution of $\mathrm{H}_{0}$ can be approximated by a $\chi^{2}$ distribution. This is particularly true if the number of observation in each group is important, if not, it is suggested to adjusting $\mathrm{H}_{0}$.

For a given confidence interval, the tested hypothesis is declared as null if its observed value is higher than the theoretical $\chi^{2}$ value adjusted to the degree of freedom.

In practice, to check if hypothesis can be considered as null and approximated by a $\chi^{2}$ distribution, a $p$-value is used. This value represents the probability of obtaining theoretical $\chi^{2}$ values that are equal or higher than the observed test statistic. A $p$-value lower than 0.05 suggests that tested hypothesis should be considered as a null one.

From averages hardness values of the three investigated samples, it is clear that distribution associated to sample SLIP is not confused $(p<0.0001)$ with distribution associated to samples which have been shaped by a pressing way. Indeed, average hardness value of SLIP sample (4.62 GPa) is lower than mean hardness values of ISOP and UNIP samples (respectively, 5.22 and $5.20 \mathrm{GPa}$ ). We may remind you that this difference of hardness is due to the difference of sintered density between samples.

It is interesting to realize Kruskall-Wallis test with the two samples which have been shaped by pressing step (UNIP, ISOP).

The $p$-value $(p=0.733>0.05)$ associated to this test reveals that UNIP and ISOP distributions come from the same distribution.

In others words, although distribution of UNIP sample is broader than the ISOP one, the shaping step do not have any influence on the global hardness value.

\section{References}

1. Nusair Khan, A., Lu, J. and Liao, H., Surf. Coat. Technol., 2003, 168, 291-299.

2. Gladysz, G. M., Schmücker, M., Chawla, K. K., Schneider, H., Joslin, D. L. and Ferber, M. K., Mater. Charact., 1998, 40, 209-214.

3. Muchtar, A. and Lim, L. C., Acta Mater, 1998, 46-5, 1683-1690.

4. Camerucci, M. A., Urretavizcaya, G. and Cavalieri, A. L., J. Eur. Ceram. Soc., 2001, 21, 1195-1204.

5. Pettersson, P., Johnsson, M. and Shen, Z., J. Eur. Ceram. Soc., 2002, 22, 1883-1889.

6. Andersson, T. and Rowcliffe, D. J., J. Am. Ceram. Soc., 1996, 79(6), 1509-1514.

7. Tancret, F. and Osterstock, F., Scripta Mater., 1997, 37(4), 443-447.

8. Nieto, M. I., Martinez, R., Mazerolles, L. and Baudin, C., J. Eur. Ceram. Soc., 2004, 24, 2293-2301.

9. Cheng, Y. T. and Cheng, C. M., Surf. Coat. Technol., 2000, 133/134, 417-424.

10. Guillaume, B., Boschini, F., Garcia-Cano, I., Rulmont, A., Cloots, R. and Ausloos, M., J. Eur. Ceram. Soc., 2005, 25(16), 3593-3604.

11. Boschini, F., Guillaume, B., Rulmont, A., Cloots, R. and Moreno, R., J. Eur. Ceram. Soc., 2006, 26(9), 1591-1598.

12. Ziemath, E. C., J. Non-Cryst. Solids, 2000, 273, 19-24.

13. Alcala, J., Acta Mater., 2000, 48, 3451-3464.

14. Zeng, K., Söderlund, E., Giannakopoulos, A. E. and Rowcliffe, D. J., Acta Metall., 1996, 44, 1127-1141.

15. Walker, W. J., Reed, J. S. and Verna, S. K., J. Am. Ceram. Soc., 1999, 82(1), 50-56.

16. Glass, S. J. and Ewsuk, K. G., Mater. Res. Soc. Bull., 1997, 22(12), 24-28.

17. Onoda, G., Presented at the 97th Annual Meeting of the American Ceramic Society, Cincinnati, OH, 1995.

18. Weibull, W., J. Appl. Mech., 1951, 293-297.

19. Gong, J., Ceram. Int., 2002, 28, 767-772.

20. Fett, T., Ernst, E., Munz, D., Badenheim, D. and Oberacker, R., J. Eur. Ceram. Soc., 2003, 23, 2031-2037.

21. Burrow, M. F., Thomas, D., Swain, M. V. and Tyas, M. J., Biomaterials, 2004, 25, 5031-5035.

22. Montgomery, D. C, Introduction to Statistical Quality Control (2nd ed.), John Wiley \& Sons, 461-465.

23. Kruskal, W. H. and Wallis, W. A., J. Am. Stat. Assoc., 1952, 47, 583-621.

24. Lehmann, E. L., Nonparametrics: Statistical Methods Based on Ranks (Facsimile edition). Pearson Education, 1998. 